\title{
Review Ariticle
}

\section{The Effects of Genetically Modified Organisms (GMO) on Environment and Molecular Techniques to Minimize Its Risk}

\author{
Girma Haile ${ }^{1,4,{ }^{*}, \text { Mulugeta Adamu }}{ }^{2}$, Tadele Tekle ${ }^{3}$ \\ ${ }^{1}$ Department of Crop Production and Landscape Management, Ebonyi State University, Abakaliki, Nigeria \\ ${ }^{2}$ Department of Biotechnology, Mekelle University, Mekelle, Ethiopia \\ ${ }^{3}$ Department of Biology, Mizan-Tepi University, Mizan-Tepi, Ethiopia \\ ${ }^{4}$ Department of Horticulture and Plant Science, Jimma University, Jimma, Ethiopia
}

\section{Email address:}

girmahaile80@gmail.com (G. Haile), mulugetaadamu06@gmail.com (M. Adamu), tadeletekle2007@gmail.com (T. Tekle)

${ }^{*}$ Corresponding author

\section{To cite this article:}

Girma Haile, Mulugeta Adamu, Tadele Tekle. The Effects of Genetically Modified Organisms (GMO) on Environment and Molecular Techniques to Minimize Its Risk. American Journal of Polymer Science and Technology. Vol. 6, No. 4, 2020, pp. 32-39.

doi: $10.11648 /$ j.ajpst.20200604.11

Received: November 13, 2020; Accepted: December 1, 2020; Published: December 11, 2020

\begin{abstract}
Biotechnology is the application of scientific techniques to modify and improve plants, animals, and microorganisms to enhance their value. Genetically modified organism (GMO) is any organism whose genetic material has been altered using genetic engineering techniques. Transgenic technology deals with the integration of exogenous DNA into the plant genome using gene transfer technologies. Genetically modified (GM) crop plants contain artificially inserted gene (s) or "transgenes" from another unrelated plant or from a completely different species via advanced genetic engineering techniques. Transgenic crops do not present new categories of environmental risk compared to conventional methods of crop improvement. Transgenic plants can be generated using (1) biolistic technique where in the desired gene is coated on to either gold or tungsten particles are shot into plant cells using a gene-gun. The necessary criterion for this is that cells or plant tissues should be suitable for transformation permit regeneration of a whole plant thereafter. (2) The other major gene transfer technique makes use of the soil bacterium Agrobacterium tumefaciens, containing a tumor-inducing (Ti) plasmid including virulence (vir) gene (s) and a transferred-DNA (T-DNA) region, in which genes of interest can be inserted. Generally the gene that transfer from other organism to the desired crop (GM) can cause different environmental risks like, generation of super weed, development of tolerance to target herbicide, loss of biodiversity and sustainable resistance in insect pests, through gene flow. These risks can be minimized using different molecular techniques such as, maternal inheritance, male sterility, cleistogamy and apomixes, genetic use restriction technologies, genome incompatibility, controlling gene expression and transgenic mitigation.
\end{abstract}

Keywords: Environment, Genetically Modified Organism, Genetics, Molecular Technique, Transgenic Crop

\section{Introduction}

Biotechnology is the application of scientific techniques to modify and improve plants, animals, and microorganisms to enhance their value. Agricultural biotechnology is the area of biotechnology involving applications to agriculture. Genetically modified organism (GMO) is any organism whose genetic material has been altered using genetic engineering techniques. It is the result of a laboratory process where genes from the DNA of one species are extracted and artificially forced into the genes of an unrelated plant or animal. The foreign genes may come from bacteria, viruses, insects, animals or even humans.

When the science of plant breeding was further developed in the 20th century, plant breeders understood better how to select superior plants and breed them to create new and improved varieties of different crops. This has dramatically increased the productivity and quality of the plants we grow for food, feed and fiber. Conventional plant breeding has been the method used to develop new varieties of crops for 
hundreds of years. However, conventional plant breeding can no longer sustain the global demand with the increasing population, decline in agricultural resources such as land and water, and the apparent plateauing of the yield curve of the staple crops. Thus, new crop improvement technologies should be developed and utilized.

In the light of mounting population pressures and rising quality-of-life expectations, food systems are challenged to meet current global needs and for the upcoming future. In undeveloped and developing countries, enormous increase in population has resulted in poverty, food insecurity, and poor nutrition among the masses. Advances in scientific discovery and laboratory techniques have led to the ability of plant improvement through the use of biotechnology and genetic engineering by manipulating existing genetic resources.

For the last decade and half, conventional crops have been genetically modified for a variety of reasons including longer shelf life, improved nutritional value, enhanced agronomic traits such as herbicide tolerance, microbial/ insect resistance, and tolerance to various severe environmental perturbances $[17,25]$. To enhance the food supply by increasing crop yields, plants are continuously being bioengineered and/or genetically engineered (GE)/genetically modified (GM) and GM plants are now prevalent worldwide and appear in many processed food products [36). In 1996, GM crops were first introduced into the commercial market in the United States and were rapidly adopted by farmers. Great success was achieved in increasing agricultural productivity to fulfill human needs during the 20th century due to the introduction of GM crops [43, 37].

GM crop plants contain artificially inserted gene (s) or "transgenes" from another unrelated plant or from a completely different species via advanced genetic engineering techniques $[35,3]$. Using various recent approaches, several varieties of GM crops have been developed and received approval for environmental release or commercial use [9].

Genetically modified (GM) plants, also called transgenic plants, are designed to acquire useful quality attributes such as insect resistance, herbicide tolerance, a biotic stress tolerance, disease resistance, high nutritional quality, high yield potential, delayed ripening, enhanced ornamental value, male sterility, and production of edible vaccines. Another major goal for raising the GM plants is their application as bioreactors for the production of nutraceuticals, therapeutic agents, antigens, monoclonal antibody fragments biopolymers, and so forth [39]. Thus, GM plants can potentially affect many aspects of modern society, including agricultural production and medical treatment. Despite these potential applications, the use of GM plants for human welfare has been restricted owing to various concerns raised by the public and the critics.

Transgenic plants can be generated using (1) biolistic technique where in the desired gene is coated on to either gold or tungsten particles are shot into plant cells using a gene-gun $[6,1]$. The necessary criterion for this is that cells or plant tissues should be suitable for transformation permit regeneration of a whole plant thereafter. (2) The other major gene transfer technique makes use of the soil bacterium
Agrobacterium tumefaciens, containing a tumor-inducing (Ti) plasmid including virulence (vir) gene (s) and a transferred-DNA (T-DNA) region, in which genes of interest can be inserted [5]. The ability of Agrobacterium species to transfer DNA to plants has been exploited by scientists for genetic engineering purposes. Once transferred, these "transgenes" endow plants with new characteristics such as herbicide or pathogen resistance, ability to produce drugs or edible vaccines, etc. The transferred constructs generally contain a promoter element that allows the regulation of transgene expression either quantitatively or in a specific manner.

Transgenic crops do not present new categories of environmental risk compared to conventional methods of crop improvement. "However, with the long-term trend toward increased capacity to introduce complex novel traits into the plants, the associated potential hazards, and risks, while not different in kind, may nonetheless be novel" [33]. The nature of the risks vary depending on the characteristics of the crop, the ecological system in which it is grown, the way it is managed, and the private and public rules governing its use. Three categories of hazard emerge from the interaction of these factors. Table 1 shows often-mentioned environmental concerns for herbicide-tolerant, virus-resistant and insect-resistant crops.

The risk of a transgene spreading in the environment is related to the likelihood for out-crossinghorizontal gene transfer, and the phenotype imparted by the gene [24]. Debates about the commercial introduction of GM plants in some parts of the world have led to questions about their potential impact on the environment unless necessary safeguards are taken into account [11] Thus, this paper aimed to review and document on the Current Molecular (Genetic) methods for minimizing risk of Genetically Modified crops on environment.

\section{Transformation Techniques Used for the Production of GM Crops}

Transgenic technology deals with the integration of exogenous DNA into the plant genome using gene transfer technologies [23], such as, agrobacterium method, bombardment, electroporation, microinjection, gene transfer by polyethylene glycol and liposome mediated gene transfer. Agrobacterium mediated gene transfer and bombardment methods are widely used for development of transgenic plants [31].

\subsection{Agrobacterium-Mediated Transformation}

It is an indirect or vector-based transformation method, and utilizes the ability of Agrobacterium tumefaciens bacteria to copy and transfer a specific portion of DNA (T-DNA) present on a tumor-inducing (Ti) plasmid into the nucleus of the plant cell. This allows for the integration of the DNA into chromosomes and subsequently leading to the integration of the T-DNA into the plant genome. This type of transformation 
involves three stages [19].

The initiation stage entails the insertion of the gene of interest into a suitable functional construct. The construct includes the gene expression promoter, gene of interest, selectable marker as well as codon modification. The initiation stage then continues to the insertion of the transgene into the Ti-plasmid. The final step of the initiation stage involves the insertion of the T-DNA, which contains the transgene, into Agrobacterium. The next stage is the bacterium-to-plant transfer during which the transformed bacteria are mixed with plant cells to facilitate the transfer of T-DNA into the plant genome.

Table 1. Selected transgenic traits and environmental concerns [45].

\begin{tabular}{ll}
\hline Genotype & Environmental concerns \\
\hline & 1. Increased weediness of wild relatives of crops through gene flow \\
Herbicide tolerance (HT) & 2. Development of HT weed populations through avoidance and selection \\
& 3. Development of HT 'volunteer' crop populations \\
& 4. Negative impact on animal populations through reduction of food supplies \\
Virus resistance (VR) & 2. Increased weediness of wild relatives of crops through gene flow \\
& 3. Development of more virulent and difficult to control viruses through virus altration \\
& 1. Increased weediness of wild relatives of crops through gene flow \\
Insect resistance (IR) & 2. Development of IR populations \\
& 3. Toxicity to non-target and beneficial insect and soil micro-organism populations
\end{tabular}

The final stage is nucleus targeting where the transgene is randomly integrated into the plant chromosome. Following nucleus targeting non-homologous end-joining processes [21] enables the integration of T-DNA into the plant genome in the absence of any homology between the T-DNA and plant DNA sequences [34]. The possible need for tissue culture steps on selective artificial media associated with Agrobacterium transformation may lead to Somaclonal variations, which in itself may lead to genetic changes in the host genome.

\subsection{Particle Bombardment}

Particle bombardment or biolistic transformation is commonly used to transform plants that are not susceptible to Agrobacterium transformation [7]. The integration of transgenes into a host plant genome, following particle bombardment, generally occurs non-randomly at AT-rich regions carrying nuclear matrix attachment region (MAR) motifs [32]. These elements have been postulated to be target sites for transgene integration into the host plant genome [32, 4].

\section{Environmental Effects of GMO}

\subsection{Transfer of Genes-Gene Flow}

There is little doubt in the scientific community that genes will move from crops into the wild $[22,18]$. The relevant research questions are whether transgenes will thrive in the wild, and how they might convey a fitness advantage to wild plants that makes them more difficult to control in areas [22, $33,40]$.

Generally, crops with wild relatives in close proximity to the areas where the crops are grown, pose higher risk for gene flow to wild relatives. In USAfor examples include sunflower (Helianthus annuus L) and oilseed rape (Brassica napus) [22, 40]. Gene transfer could become a problem if the transferred genes do not have deleterious effects on the crop-wild hybrids, but instead confer an ecological advantage [22, 40]. Gene flow from classically bred crops to wild plants has been documented. [16] finds that classically bred crop-to-wild gene flow has enhanced the 'weediness' of weeds for seven of the world's thirteen most important crops (e.g. Johnson grass (Sorghum halepense) from cultivated sorghum (Sorghum bicolor)).

\subsubsection{Transgene Escape to Wild-type Plants}

There is a potential risk that the GM plants may hybridize (or cross-breed) with sexually compatible wild-type species [40]. This genetic exchange is possible due to wind pollination, biotic pollination or seed dispersal. This may have an impact on the environment through the production of hybrids and their progeny.

\subsubsection{Generation of Super Weeds}

The concern of gene flow from GM plants to weedy relatives via pollination is quite intense [24]. It is considered that the transfer of encoded characteristics to weed species could potentially give them a selective advantage, consequently leading to the generation of "super weeds." Moreover, the newly introduced traits may make a plant, especially herbicide tolerant plant, more persistent or invasive (weedy) in agricultural habitats [30].

\subsubsection{Development of Tolerance to Target Herbicide}

It is viewed that the repeated use of the same herbicide in the same area to remove weeds amongst genetically modified herbicide resistant crops (HRCs) (tolerant to single herbicide) will exacerbate the problem of herbicide-tolerant weeds [24]. Another matter of concern relates to the plants carrying different herbicide tolerance genes to become multiply tolerant to several herbicides by pollination between adjacent plants [41].

\subsubsection{Sustainable Resistance in Insect Pests}

It is possible that the widespread use of disease-resistant GM plants may lead to the evolution of several insect pests that are resistant to pesticides [13]. For example, Btcrops may develop resistance to $B$ tbiopesticide, a permitted biopesticide 
successfully used by organic farmers in the integrated pest management (IPM) programs. There is to date no reported evidence of insect resistance to Btcrops under field conditions although Btresistant insects (e.g., cotton budworm and bollworm) have been observed in areas where Btbiopesticides are sprayed on crops [2].

\subsubsection{Loss of Biodiversity}

The public has long been worried about the loss of plant biodiversity due to global industrialization, urbanization, and the popularity of conventionally-bred high-yielding varieties. It is speculated that the biodiversity will be further threatened due to the encouraging use of GM plants. This is because development of GM plants may favor monocultures, that is, plants of a single kind, which are best suitable for one or other conditions or produce one product [24]. Further, the transformation of more natural ecosystems into agricultural lands for planting GM plants is adding to this ecological instability.

\subsection{Impacts of GMO on Non-target Organisms}

Whereas crops bred to resist pests may endure less damage and lead farmers to use less insecticide, there is concern that the toxins these plants produce may harm non-target organisms, including animals and plants that are not pests. Laboratory research confirms that transgenic insecticidal crops can have negative impacts on potentially beneficial non-target organisms, including lacewings. Recent farm trials in the UK confirm that enhanced weed-control efficacy from using herbicide-tolerant crops can reduce food supplies and lower the populations of non-target species such as bees, butterflies and seed-eating beetles. However the results varied considerably by the type of herbicide employed in the system. Additional findings from the data collected from these trials should be forthcoming and help to shed further light on these complex interactions [45].

\section{Molecular Techniques to Minimize Risk of GM Crops on Environment}

There are several containment methods currently in use to reduce the likelihood of gene flow occurring. The majority of containment strategies is the use of genetic strategies, which are less dependent on human oversight [28]. Some of these techniques are discussed below.

\subsection{Maternal Inheritance}

Maternal inheritance of cytoplasmic organelles is shared by plant chloroplasts. It promotes the invasion of a population by selfish cytoplasmic factors that are overrepresented within an individual [12]. In addition, maternal inheritance of cytoplasmic factors is an evolutionary mechanism to prevent sexual transmission of disorders or pathogens associated with males; only the nucleus (not cytoplasm) is allowed to penetrate the ovule during fertilization [20]. It may also be an extension of the general suppression of male nuclear genes that takes place in plants after fertilization [42]. The use of chloroplast genetic engineering to promote maternal inheritance of transgenes is highly desirable in those instances involving a potential for outcross among GM crops or between GM crops and weeds. The prevalent pattern of plastid inheritance found in the majority of angiosperms is uniparental-maternal and chloroplast genomes are maternally inherited in most crops [12].

Maternal inheritance of the chloroplast genome is achieved in plants during the development of the generative cells that form sperm cells, which then fuse with the female gametes during fertilization. The generative cells are the result of unequal divisions during pollen formation and do not receive any chloroplasts [12]. In some species, chloroplast DNA is degraded during generative and sperm cell formation, resulting in physical exclusion of chloroplast DNA during sexual fusion. Maternal inheritance of transgenes and prevention of gene flow through pollen in chloroplast transgenic plants have been successfully demonstrated in several plant species [38]. Generally, the chloroplast transgenic lines were fertile, flowered and set seeds similar to untransformed plants. Transgenes stably integrated into the cotton chloroplast genome were maternally inherited and were not transmitted via pollen when out-crossed with untransformed female plants. Cotton is one of the most important genetically modified crops. Successful transformation of the chloroplast genome should address concerns about transgene escape, insects developing resistance, inadequate insect control and promote public acceptance of genetically modified cotton [26].

\subsection{Male Sterility}

Male sterility in plants implies an inability to produce or to release functional pollen, and is the result of failure of formation or development of functional stamens, microspores or gametes [27]. Cytoplasmic male sterility is one that included under this technique. Cytoplasmic male sterility (CMS), a condition under which a plant is unable to produce functional pollen, is widespread among higher plants [15]. Many male-sterility mutations interfere with tapetal cell differentiation and/or function, indicating that this tissue is essential for the production of functional pollen. It was exploited, using the 5 ' region of a tobacco tapetum-specific gene (TA29) to drive expression of recombinant $\beta$-glucuronidase or ribonuclease genes (RNase $\mathrm{T} 1$ and barnase) within the tapetal cells of transgenic tobacco and oilseed rape plants. Expression of RNase genes selectively destroys the tapetum during anther development, preventing pollen formation and producing male-sterile plants [12].

Male sterility is also the basis of the BarstarBarnase system used in the Plant Genetic Systems (Ghent, Belgium) glufosinate (Bar-gene)-tolerant rapeseed. In this system, cauliflower mosaic virus (CaMV) 35S promoter-directed expression of the ribonucleasebarnase from Bacillus amyloliquefaciens inhibits pollen formation and results in 
male sterility of the transformed plants. Linkage of the barnase gene with the (Streptomyceshygroscopicus) bar marker gene, which encodes a phosphinothricinacetyltransferase enzyme that inactivates glufosinate, permits identification of the male-sterile line before crops begins to flower. GM rapeseed containing the BarstarBarnase system compose $\sim 10 \%$ of the commercially cultivated crop in Canada and is one of the few GMOs cleared for agricultural use in Europe [12].

These two examples suggest that male sterility, induced by destruction of the tapetum by transgene-encoded ribonucleases, could allow gene containment. However, while interfering with pollen development may be effective in preventing gene flow in many cases, under exceptional circumstances it is possible that a GM crop engineered to be male-sterile could be fertilized by pollen from wild relatives and serve as a female parent for hybrid seed. If this hybrid were to survive, germinate, grow, and reproduce, it could produce viable pollen containing the GM trait that could cross-pollinate weeds [12].

\subsection{Cleistogamy and Apomixes}

Apomixes (asexual reproduction through seeds) and Cleistogamy (self pollination and Fertilization of an unopened flower) have been proposed as a possible method by which to contain transgenes in genetically modified (GM) crops [10]. In certain plants, self-pollination and fertilization occurs with the flowers remaining unopened a process termed cleistogamy. It has been suggested that crops engineered to exhibit cleistogamy would have a minimal risk of gene spread ([12]. Several genes are involved in flower development have recently been identified in model plants. An additional limitation of this approach is that even if cleistogamy could be engineered into certain crops carrying a GM trait, it might prove ineffective. In rice that exhibits cleistogamy, for example, genes readily move between cultivated and feral forms of weedy rice, despite predominant self-pollination [12].

Apomixes occurs naturally in a few plant species. In apomixes, the seed is actually of vegetative origin and not produced from sexual pollination. Because many apomictic plants produce no viable or compatible pollen, it is possible that the method could be used to create GM plants with reduced risk of gene transfer without compromising seed or fruit production. The apomictic embryo is formed in the ovule through sporophytic (from the integument or nucellus) or gametophytic (from the megaspore mother cells or nuclear cells) pathways. Irrespective of origin, generation of fertile seeds can be dependent on fertilization for the formation of endosperm. However, autonomous apomicts develop an endosperm independently of fertilization of polar nuclei. Apomixes fixes a maternal genotype, because the male gametophyte makes no contribution to the genetic makeup of the embryo and meiosis is not necessary. Therefore, in addition to gene containment, this process is very helpful to fix a superior plant variety [12].

\subsection{Genetic Use Restriction Technologies}

Genetic use restriction technologies (GURTs), developed to secure return on investments through protection of plant varieties, are among the most controversial and opposed genetic engineering biotechnologies as they are perceived as a tool to force farmers to depend on multinational corporations' seed monopolies. Variety-GURT (also known as suicide/sterile seed/gene technology, or terminator technology) is designed to control plant fertility or seed development through a genetic process triggered by a chemical inducer that will allow the plant to grow and to form seeds, but will cause the embryo of each of those seeds to produce a cell toxin that will prevent its germination if replanted, thus causing second generation seeds to be sterile and allowing manufacturers to maintain their intellectual property rights and avoid concerns related to GM seed dispersal [29].

Genetic use restriction technologies could be used for the environmental containment of transgenic seeds (V-GURT) or transgenes (T-GURT), thus solving or marginalizing one of the greatest concerns associated with GM crops. Seed lethality is the only strategy at present that prevents transgene movement via seeds [14]. However; GURTs may generally prevent unwanted gene flow from transgenic to non-transgenic varieties (including wild relatives) because it is argued that pollen carries the dominant allele of the lethal/inhibiting protein. Thus, the GURT would most likely be transferred along with the desired trait in the hybrid through cross-pollination [28].

\subsection{Genome Incompatibility}

Many cultivated crops have multiple genomes. Only one of these crop genomes is compatible for interspecific hybridization with weeds. For example, the D genome of wheat is compatible with the D genome of Aegilopscylindrica (bearded goatgrass), a problem weed in the United States; in contrast, it would be much harder to achieve interspecific hybridization of the weed with durum wheat, which has an AABB tetraploid B genome [20], provided ploidy level is not an issue. Similarly, there is possibility for gene transfer from the B genome of Brassica juncea (Indian or brown mustard) to many Brassica weeds with wild species; however, thus far most genetic engineering has been carried out Brassica napus, which has the AACC tetraploid genome and is thus unlikely to be compatible. The risk of transgenic traits spreading into weeds can be reduced drastically by releasing only those transgenic lines with incompatible genomes. However, the approach is not likely to work in all crops.

In general, much more information is required to determine the genes responsible for compatibility of weeds and specific crops. With the availability of such information, it might become possible to engineer crops that have a reduced likelihood of out crossing with weeds through incompatibility mechanisms. It will also be important to assess the fertilities of interspecific and intraspecific hybrids of crops and weeds on a case-by-case basis. Of course, although genome compatibility approaches might provide a solution to gene flow from a GM 
crop, they also raise the problem of how one would cross-pollinate the GM crop itself if no sexually compatible weed or related crop were available nearby [12].

\subsection{Controlling Gene Expression}

The exposure of non-target organisms to recombinant proteins can be minimized by restricting expression to particular tissues. For example, a number of promoters have been identified that restrict gene expression to seeds or fruit. This would prevent the consumption of the protein by insects and other animals feeding on green plant tissue and would likewise avoid other forms of contact, such as the exposure of pollinating insects to recombinant proteins expressed in pollen grains. By avoiding transgene expression in roots, leaching of the recombinant protein into the soil (and consequent disruption of the rhizosphere) would also be prevented. If restricted expression strategies were used in combination with effective management (e.g. specific harvesting times) then vegetative transgenic material could safely decay in the environment with no risk of protein toxicity [8]. An alternative strategy is to bring the transgene under inducible control, such that the recombinant protein would be expressed only when the plant was exposed to a certain chemical inducer [44].

\subsection{Transgenic Mitigation}

Another approach for containing gene spread would be to compromise the fitness of weeds that by introgression have acquired positive survival traits from crop genes. This approach, termed transgenic mitigation (TM), is based on the premises that (1) tandem constructs act as tightly linked genes, and their segregation from each other is exceedingly rare; (2) TM traits are neutral or positive for crops, but deleterious for weeds; and (3) even mildly harmful TM traits will be eliminated from weed populations because such plants compete strongly among themselves and have a large seed output. Examples of processes that might be targeted by TM include seed dormancy, seed ripening and shattering, and growth [12].

\section{Conclusion}

GM crop plants contain artificially inserted gene (transgenes) from another unrelated plant or from a completely different species via advanced genetic engineering techniques. They are modified for benefits like, insect resistance, herbicide tolerance, abiotic stress tolerance, disease resistance, high nutritional quality, high yield potential, delayed ripening, etc. However, the gene that transfer from other organism to the desired crop (GM) can cause different environmental risks like, generation of super weed, development of tolerance to target herbicide, loss of biodiversity and sustainable resistance in insect pests, through gene flow.

Impacts of GMO on non-target organisms whereas crops bred to resist pests may endure less damage and lead farmers to use less insecticide, there is concern that the toxins these plants produce may harm non-target organisms, including animals and plants that are not pests. These risks can be minimized using different molecular techniques such as, maternal inheritance, male sterility, cleistogamy and apomixes, genetic use restriction technologies, genome incompatibility, controlling gene expression and transgenic mitigation. Specifically, advances in genetic engineering have made possible the manipulation of crops to increase yield, guaranteeing food supplies for the increasing world population [47]. Globally, today genetically modified crops are grown in fields on a commercial scale. Thus, the biotech crop area has increased from 1.7 million ha in 1996 to 189.8 million ha in 2017 [46, 47]. Generally, environmental effects of GMO should be minimized by using molecular techniques to insure food security in the world.

\section{Conflicts of Interest}

All authors declare no conflicts.

\section{References}

[1] Allen, G. C., Spiker, S. and Thompson, W. F., 2005. Transgene integration: use of matrix attachment regions. In Transgenic Plants: Methods and Protocols (pp. 313-326). Humana Press.

[2] Alstad, D. N. and Andow, D. A., 1995. Managing the evolution of insect resistance to transgenic plants. Science, 268 (5219), pp. 1894-1896.

[3] Bock, R. and Khan, M. S., 2004. Taming plastids for a green future. TRENDS in Biotechnology, 22 (6), pp. 311-318.

[4] Bode, J., Benham, C., Knopp, A. and Mielke, C., 2000. Transcriptional augmentation: modulation of gene expression by scaffold/matrix-attached regions (S/MAR elements). Critical reviews in eukaryotic gene expression, 10 (1), pp. $73-90$

[5] Broothaerts, W., Mitchell, H. J., Weir, B., Kaines, S., Smith, L. M., Yang, W., Mayer, J. E., Roa-Rodriguez, C. and Jefferson, R. A., 2005. Gene transfer to plants by diverse species of bacteria. Nature, 433 (7026), p. 629.

[6] Cho, M. J., Yano, H., Okamoto, D., Kim, H. K., Jung, H. R., Newcomb, K., Le, V. K., Yoo, H. S., Langham, R., Buchanan, B. B. and Lemaux, P. G., 2004. Stable transformation of rice (Oryza sativa L.) via microprojectile bombardment of highly regenerative, green tissues derived from mature seed. Plant cell reports, 22 (7), pp. 483-489.

[7] Christou, P., 1995. Strategies for variety-independent genetic transformation of important cereals, legumes and woody species utilizing particle bombardment. Euphytica, 85 (1-3), pp. 13-27.

[8] Commandeur, U., Twyman, R. M. and Fischer, R., 2003. The biosafety of molecular farming in plants. AgBiotechNet, 5 (110), pp. 1-9.

[9] Conner, A. J., Glare, T. R. and Nap, J. P., 2003. The release of genetically modified crops into the environment: Part II. Overview of ecological risk assessment. The Plant Journal, 33 (1), pp. 19-46. 
[10] Curtis, M. D., Brand, L., Yang, W., Nüesch, E., Moore, J. M., Jefferson, R. and Grossniklaus, U., 2004, September. Apomixis technology development: Transgene containment and fixation of heterosis. In 8th International Symposium on the Biosafety of Genetically Modified Organisms. Montpellier (pp. 167-173).

[11] Dale, P. J., Clarke, B. and Fontes, E. M., 2002. Potential for the environmental impact of transgenic crops. Nature biotechnology, 20 (6), p. 567.

[12] Daniell, H., 2002. Molecular strategies for gene containment in transgenic crops. Nature biotechnology, 20 (6), p. 581.

[13] Dove, A., 2001. Survey raises concerns about Bt resistance management.

[14] Dunwell, J. and Ford, C. S., 2005. Technologies for biological containment of GM and non-GM crops.

[15] Eckardt, N. A., 2006. Cytoplasmic male sterility and fertility restoration.

[16] Ellstrand, N. C., 2001. When transgenes wander, should we worry? Plant physiology, 125 (4), pp. 1543-1545.

[17] Engel, K. H., Frenzel, T. and Miller, A., 2002. Current and future benefits from the use of GM technology in food production. Toxicology letters, 127 (1-3), pp. 329-336.

[18] Esnayra, J., Pool, R. and National Research Council, 2000. Ecological monitoring of genetically modified crops: a workshop summary. National Academies Press.

[19] Gelvin, S. B., 1998. The introduction and expression of transgenes in plants. Current Opinion in Biotechnology, 9 (2), pp. 227-232.

[20] Gressel, J., 2002. Molecular biology of weed control. CRC Press.

[21] Haber, J. E., 2000. Recombination: a frank view of exchanges and vice versa. Current opinion in cell biology, 12 (3), pp. 286-292.

[22] Hails, R. S., 2000. Genetically modified plants-the debate continues. Trends in Ecology \& Evolution, 15 (1), pp. 14-18.

[23] Hansen, G. and Wright, M. S., 1999. Recent advances in the transformation of plants. Trends in plant science, 4 (6), pp. 226-231.

[24] James, R. R., DiFazio, S. P., Brunner, A. M. and Strauss, S. H., 1998. Environmental effects of genetically engineered woody biomass crops. Biomass and Bioenergy, 14 (4), pp. 403-414.

[25] Konig, A., Cockburn, A., Crevel, R. W. R., Debruyne, E., Grafstroem, R., Hammerling, U., Kimber, I., Knudsen, I., Kuiper, H. A., Peijnenburg, A. A. C. M. and Penninks, A. H., 2004. Assessment of the safety of foods derived from genetically modified (GM) crops. Food and Chemical Toxicology, 42 (7), pp. 1047-1088.

[26] Kumar, S., Dhingra, A. and Daniell, H., 2004. Stable transformation of the cotton plastid genome and maternal inheritance of transgenes. Plant molecular biology, 56 (2), pp. 203-216.

[27] Lasa J. M., Bosemark N. O., 1993. Male sterility. In: Hayward M. D., Bosemark N. O., Romagosa I., Cerezo M. (eds) Plant Breeding. Plant Breeding Series. Springer, Dordrecht

[28] Lee, D. and Natesan, E., 2006. Evaluating genetic containment strategies for transgenic plants. Trends in biotechnology, 24 (3), pp. 109-114.

[29] Lombardo, L., 2014. Genetic use restriction technologies: a review. Plant biotechnology journal, 12 (8), pp. 995-1005.

[30] Manasse, R. and Kareiva, P., 1991. Quantifying the spread of recombinant genes and organisms. Biotechnology (USA).

[31] Mayee, C. D., Singh, P., Dongre, A. B., Rao, M. R. K. and Raj, S., 2003. Transgenic Bt Cotton.

[32] Morikawa, H., Sakamoto, A., Hokazono, H., IRIFUNE, K. and TAKAHASHI, M., 2002. Mechanism of transgene integration into a host genome by particle bombardment. Plant biotechnology, 19 (4), pp. 219-228.

[33] National Research Council, 2002. Environmental effects of transgenic plants: the scope and adequacy of regulation. National Academies Press.

[34] Ohba, T., Yoshioka, Y., Machida, C. and Machida, Y., 1995. DNA rearrangement associated with the integration of T-DNA in tobacco: an example for multiple duplications of DNA around the integration target. The Plant Journal, 7 (1), pp. $157-164$.

[35] Pereira, A., 2000. A transgenic perspective on plant functional genomics. Transgenic research, 9 (4-5), pp. 245-260.

[36] Perr, H. A., 2002. Children and genetically engineered food: potentials and problems. Journal of pediatric gastroenterology and nutrition, 35 (4), pp. 475-486.

[37] Rommens, C. M., Humara, J. M., Ye, J., Yan, H., Richael, C., Zhang, L., Perry, R. and Swords, K., 2004. Crop improvement through modification of the plant's own genome. Plant Physiology, 135 (1), pp. 421-431.

[38] Ruf, S., Hermann, M., Berger, I. J., Carrer, H. and Bock, R., 2001. Stable genetic transformation of tomato plastids and expression of a foreign protein in fruit. Nature biotechnology, 19 (9), p. 870.

[39] S. Rastogi and N. Pathak, 2009. Genetic Engineering, Oxford University Press, New Delhi, India.

[40] Snow, A. A. and Palma, P. M., 1997. Commercialization of transgenic plants: potential ecological risks. BioScience, 47 (2), pp. 86-96.

[41] Traynor, P. L. and Westwood, J. H., 1999, January. Ecological effects of plant resistance genes in managed ecosystems. In Workshop Proceedings, Information Systems for Biotechnology (Vol. 31).

[42] Vielle-Calzada, J. P., Baskar, R. and Grossniklaus, U., 2000. Delayed activation of the paternal genome during seed development. Nature, 404 (6773), p. 91.

[43] Yan, L. and Kerr, P. S., 2002. Genetically engineered crops: their potential use for improvement of human nutrition. Nutrition Reviews, 60 (5), pp. 135-141.

[44] Zuo, J. and Chua, N. H., 2000. Chemical-inducible systems for regulated expression of plant genes. Current Opinion in Biotechnology, 11 (2), pp. 146-151.

[45] Ervin, D. E. and Welsh, R., 2006. Environmental effects of genetically modified crops: differentiated risk assessment and management. In Regulating agricultural biotechnology: Economics and policy (pp. 301-326). Springer, Boston, MA. 
[46] Briefs, I. S. A. A. A., 2017. Global status of commercialized biotech/GM crops in 2017: Biotech crop adoption surges as economic benefits accumulate in 22 years.
[47] Babiye, B., Haile, G. and Adamu, M., 2020. Major Achievements of Plant Biotechnology in Crop Improvements. American Journal of Life Sciences, 8 (5), pp. 102-106. 